Pradas de la Fuente, F.; González-Jurado, J.A.; García-Giménez, A.; Gallego Tobón, F. y Castellar Otín, C. (2019) Características antropométricas, de jugadores de pádel de élite. Estudio piloto / Anthropometric Characteristics of Elite Paddle Players. Pilot Study. Revista Internacional de Medicina y Ciencias de la Actividad Física y el Deporte vol. 19 (74) pp. 181-195 Http://cdeporte.rediris.es/revista/revista74/artcaracteristicas1012.htm

DOI: http://doi.org/10.15366/rimcafd2019.74.001

\title{
ORIGINAL
}

\section{CARACTERÍSTICAS ANTROPOMÉTRICAS, DE JUGADORES DE PÁDEL DE ÉLITE. ESTUDIO PILOTO}

\section{ANTHROPOMETRIC CHARACTERISTICS OF ELITE PADDLE PLAYERS. PILOT STUDY} Pradas de la Fuente, F.'; González-Jurado, J.A. ${ }^{2}$; García-Giménez, A. ${ }^{3}$;
Gallego Tobón, F. ${ }^{4}$ y Castellar Otín, C. ${ }^{5}$

\footnotetext{
1 Profesor titular de universidad, Facultad de Ciencias Humanas y de la Educación, Universidad de Zaragoza (España) franprad@unizar.es

2 Profesor titular de universidad, Facultad de Ciencias del Deporte, Universidad Pablo de Olavide (España) jagonjur@upo.es

${ }^{3}$ Doctorando en Ciencias de la Salud y del Deporte, Facultad de Ciencias Humanas y de la Educación, Universidad de Zaragoza (España) garciagimenezalejandro@gmail.com

${ }^{4}$ Magister, Facultad de Educación, Universidad San Buenaventura de Medellín (Colombia) fabian.gallego@usbmed.edu.co

5 Profesor contratado doctor, Facultad de Ciencias Humanas y de la Educación, Universidad de Zaragoza (España) castella@unizar.es
}

\section{FINANCIACIÓN}

Instituto de Estudios Altoaragoneses por el proyecto "Análisis de las demandas fisiológicas del pádel de competición: estudio de marcadores asociados al rendimiento físico-deportivo."

Código UNESCO / UNESCO code: 2402 Antropología (Física) / Anthropology (Physical)

Clasificación Consejo de Europa / Council of Europe classification: 9. Cinantropometría / Kineanthropometry

Recibido 10 de mayo de 2017 Received May 10, 2017

Aceptado 8 de abril de 2018 Accepted April 8, 2018

\section{RESUMEN}

El objetivo de este estudio fue describir las características antropométricas, la composición corporal y el somatotipo de una muestra internacional de jugadores de pádel de alto nivel de ambos sexos. En el estudio participaron 29 jugadores (15 varones y 14 mujeres) de categoría absoluta. Un total de 16 
variables antropométricas fueron evaluadas. Se encontraron diferencias entre sexos en las variables masa, talla e IMC $(p<0,001)$; en los pliegues tricipital, muslo, pierna $(p<0,001)$ y en el sumatorio de 6 pliegues; en los perímetros brazo, muslo $(p<0,001)$ y pierna $(p<0,03)$; y en todos los diámetros analizados $(p<0,001)$. Asimismo se encontraron diferencias en los componentes endomórfico $(p<0,01)$, mesomórfico $(p<0,001)$ y ectomórfico $(p<0,05)$ del somatotipo. Los jugadores presentan un somatotipo mesomórfico-endomórfico y las jugadoras endo-mesomórfico. Esta investigación aporta datos biotipológicos actualizados de referencia para el pádel de élite.

PALABRAS CLAVE: pádel; antropometría; composición corporal; somatotipo.

\section{ABSTRACT}

Paddle is one of the racket sports that has grown the most in recent years. However, there are few or very limited studies that address the biotype of this discipline, especially in the elite paddle. The aim was to describe the anthropometric characteristics, body composition and somatotype of an international sample of high level paddle players. 29 subjects national top level (15 male and 14 female) participated in this study. 16 anthropometric variables were evaluated. Differences were found between sexes in the variables weight, height and BMI $(p<0.001)$; in triceps, thigh and leg folds $(p<0.001)$; in the arm, thigh $(p<0.001)$ and leg $(p<0.03)$ perimeters; and in all diameters analysed ( $p$ $<0.001)$. Differences were also found between men and women in the endomorphic $(p<0.01)$, mesomorphic $(p<0.001)$ and ectomorphic $(p<0.05)$ components of the somatotype. Male players present a mesomorphicendomorphic somatotype while female players are preferably endomesomorphic. This research provides up-to-date reference data for somatotype in elite paddle players.

KEY WORDS: paddle; body composition; anthropometry; somatotype.

\section{INTRODUCCIÓN}

El carácter del esfuerzo que se produce durante la práctica del pádel es de tipo acíclico, sucediéndose continuamente periodos intermitentes de trabajo y descanso de manera similar a otras modalidades de raqueta y pala (CabelloManrique \& Gonzalez-Badillo, 2003; Chin, Steininger, So, Clark, \& Wong, 1995; Fernández, Mendez-Villanueva, \& Pluim, 2006; Pradas, González-Jurado, Molina Sotomayor, \& Castellar Otín, 2013). Este deporte se practica en parejas y se desarrolla en un área de juego rectangular dividida en dos campos mediante una red central. La pista de pádel se caracteriza por encontrarse su perímetro totalmente cerrado, combinándose zonas de malla metálica y zonas de materiales que permiten un rebote regular de la pelota (paredes de fondo y laterales) contra las que se permite su juego. 
La singularidad de la práctica del pádel respecto a otros deportes de raqueta y pala radica en su exclusiva dinámica de juego, siendo la única disciplina en la que se permite devolver la pelota enviada por la pareja rival mediante tres formas diferentes: a) de manera directa golpeando la pelota hacia el campo contrario sin dejarla botar o tras un bote sobre la pista; b) realizando el golpeo tras rebotar la pelota sobre el terreno de juego y a continuación alguna pared (lateral, de fondo o ambas); c) De manera indirecta golpeando la pelota contra las propias paredes laterales o de fondo de la zona de juego en donde los jugadores se encuentran situados (Pradas et al., 2014)

Desde el punto de vista fisiológico el pádel tiene la particularidad de reunir la participación de las diferentes rutas metabólicas. Atendiendo a la estructura temporal de las diferentes acciones de juego que se producen en este deporte, se aprecia un predominio del sistema metabólico anaeróbico láctico, aunque en menor medida también existe una actividad anaeróbica aláctica. No obstante, como consecuencia de la duración total de los partidos se hace necesaria también la intervención del metabolismo aeróbico, siendo considerado en este deporte el sistema más importante de soporte energético (Castellar, Pradas, Quintas, Arraco, \& Pérez, 2015).

La intensidad desarrollada durante la práctica del pádel se aproxima a la experimentada en la modalidad individual del tenis. Sin embargo, a pesar de que el consumo de oxígeno de los jugadores de pádel es similar al alcanzado por los de tenis, tanto en valores máximos como relativos, las exigencias reales de cada práctica son diferentes (de Hoyo, Sañudo, \& Carrasco, 2007).

El análisis del biotipo que caracteriza a los jugadores es otro de los aspectos importantes a considerar por su relación directa con el rendimiento deportivo. Si nos centramos en los deportes de raqueta y pala se puede comprobar que son numerosos los estudios que se preocupan por determinar con precisión las características antropométricas en disciplinas como el squash (Mellor et al., 1995), el bádminton (Vicén, Abián-Vicén, \& Sampedro, 2012), el tenis (Sánchez-Muñoz, Sanz, \& Zabala, 2007) o el tenis de mesa (Pradas et al., 2013; Sepúlveda, Barraza, Soto, Báez, \& Tuesta, 2015).

Sin embargo, a pesar de que en la actualidad existen interesantes investigaciones que intentan describir el perfil antropométrico en el pádel, resulta ciertamente difícil poder estandarizar los datos que se presentan por su heterogeneidad, ya que en algunos casos los estudios han sido realizados con deportistas universitarios (Martinez-Rodriguez, Collado, \& Vicente-Salar, 2015), con muestras de elite muy reducidas (Pradas et al., 2014), e incluso comparando jugadores de diferentes niveles de juego (Castillo-Rodríguez, Hernández-Mendo, \& Alvero-Cruz, 2014).

Por otro lado, el importante impulso, desarrollo y profesionalización que ha sufrido el pádel en los últimos años, ha supuesto una importante reducción del número de jugadores que practicaban esta disciplina provenientes de otros 
deportes de raqueta y pala, en especial del tenis, aumentando paulatinamente los deportistas que se han especializado únicamente en este deporte.

La incorporación a la élite en los últimos años de jugadores formados exclusivamente desde edades tempranas en este deporte, permite a los investigadores aproximarse al conocimiento de un biotipo de referencia más específico, cercano y acorde a la realidad deportiva del pádel de alto nivel que se practica en estos momentos.

Considerando lo expuesto hasta el momento, determinar las características antropométricas y la composición corporal que caracteriza en la actualidad a esta disciplina, podría resultar de interés por su relevancia para obtener un adecuado rendimiento deportivo. En este sentido, el objetivo de este estudio se centra en describir y comparar el perfil antropométrico, la composición corporal y el somatotipo de jugadores de pádel de alto nivel de ambos sexos.

\section{MATERIAL Y MÉTODOS}

\section{PARTICIPANTES}

Una muestra internacional de 29 jugadores de pádel de élite, 15 hombres (edad: $28,2 \pm 7,9$ años) y 14 mujeres (edad: $29,7 \pm 3,7$ años), participaron de manera voluntaria en este estudio. Los deportistas seleccionados tenían una experiencia de 7,6 $\pm 3,3$ años y entrenaban un promedio de $10 \pm 2,8$ horas por semana en pista, participando de manera regular durante los últimos diez años en competiciones de máximo nivel de carácter nacional, en torneos Pádel Pro Tour y en pruebas del circuito World Pádel Tour.

\section{PROCEDIMIENTO}

El muestreo utilizado en esta investigación fue de tipo no probabilístico por conveniencia. Para conocer las posibles diferencias existentes entre sexos la muestra fue dividida en dos grupos: hombres y mujeres. Las medidas realizadas para las determinaciones antropométricas incluyeron: masa corporal $(\mathrm{kg})$, altura (cm), ocho pliegues (bíceps, tríceps, subescapular, suprailíaco, supraespinal, abdominal, muslo y pierna $(\mathrm{mm})$ ), cuatro perímetros (brazo relajado, brazo contraído y flexionado, muslo y pierna $(\mathrm{cm})$ ) y tres diámetros (bicondíleo femoral, biepicondíleo de húmero y biestiloideo radiolunar $(\mathrm{cm})$ ).

Para el registro de todas las medidas se utilizaron los protocolos propuestos por la International Society for the Advancement of Anthropometry (ISAK) y las recomendaciones del Grupo Español de Cineantropometría (GREC) para determinar el perfil antropométrico. Todas las mediciones fueron realizadas por un experto acreditado por la ISAK ayudado por un asistente para registrar los valores obtenidos. Para asegurar la validez y fiabilidad durante el proceso se siguieron las recomendaciones para mantener un error técnico de medida interevaluador e intraevaluador inferior a un $5 \%$ en los pliegues cutáneos y menor al $2 \%$ en el resto de mediciones. 
Para la medición de los pliegues se utilizó un plicómetro (Holtain Ltd, Crymych, UK) con una precisión de 0,2 $\mathrm{mm}$. La altura y la masa corporal fueron medidas con una báscula modelo Seca 714 (Seca Instruments Ltd, Hamburg, Germany). Los diámetros fueron evaluados con un paquímetro y los perímetros con una cinta metálica flexible con precisión de $1 \mathrm{~mm}$ (Holtain Ltd, Crymych, UK) utilizándose un lápiz dermográfico.

A partir de las evaluaciones realizadas se calcularon diferentes índices y porcentajes con el fin de determinar el índice de masa corporal (IMC), la composición corporal y el somatotipo de los deportistas participantes en el estudio. EI IMC fue calculado mediante la fórmula: [masa $(\mathrm{kg}) \mathrm{x}$ altura $(\mathrm{m})]^{2}$. La composición corporal fue estimada usando un modelo tetracompartimental, determinándose los componentes graso, óseo y muscular. El porcentaje de grasa corporal fue calculado utilizando la fórmula de Withers, Craig, Bourdon \& Norton (Withers, Craig, Bourdon, \& Norton, 1987). El análisis del somatotipo se realizó utilizando el método propuesto por Heath-Carter (Carter and Heath, 1990) obteniéndose el valor de los tres componentes: endomórfico, mesomórfico y ectomórfico.

Antes de iniciarse el estudio los deportistas fueron informados de manera verbal del objetivo, procedimiento a seguir así como de los riesgos y beneficios de su participación. Todos los deportistas firmaron un consentimiento informado por escrito. La investigación fue aprobada por el Comité Ético de Investigación Clínica del Gobierno de Aragón (España), realizándose siguiendo las directrices de la declaración de Helsinki.

\section{ANÁLISIS ESTADÍSTICO DE LOS DATOS}

El software estadístico utilizado fue el programa SPSS ${ }^{\odot}$ versión 22.0 para Windows (Inc, Chicago, Illinois). Se realizó una estadística descriptiva para obtener las medidas de tendencia central: media, desviación estándar (DE), mínimo y máximo. Para comprobar las posibles diferencias entre grupos se realizaron diferentes contrastes de hipótesis, comprobando la normalidad de la muestra mediante la prueba de Shapiro-Wilk y la homocedasticidad de varianzas a través de la prueba de Levene. Cuando las muestras cumplían con las condiciones de normalidad y homocedasticidad se utilizó la prueba T de Student para datos independientes, en su defecto se aplicó la prueba no paramétrica de U de Mann-Whitney. Para todas las comparaciones se estableció un intervalo de confianza del $95 \%(p \leq 0,05)$.

\section{RESULTADOS}

Las características generales de los jugadores que han participado en el estudio se reflejan de forma diferenciada por sexo en la Tabla 1. La muestra masculina presenta valores significativamente más elevados que la femenina en las variables biométricas masa corporal $(p<0,001)$, talla $(p<0,001)$ e IMC $(\mathrm{p}<0,001)$. 
Rev.int.med.cienc.act.fís.deporte - vol. 19 - número 74 - ISSN: 1577-0354

Tabla 1. Comparación de características generales según sexo.

\begin{tabular}{lccccccc}
\hline \multirow{2}{*}{ Variable } & \multicolumn{2}{c}{ Varones } & & \multicolumn{2}{c}{ Mujeres } & p \\
\cline { 2 - 3 } & Media $( \pm D E)$ & Rango & & Media $( \pm D E)$ & Rango & valor \\
\cline { 1 - 2 } Edad (años) & $28,2( \pm 7,9)$ & $19-46$ & & $29,7( \pm 3,7)$ & $24-37$ & $0,526^{*}$ \\
Masa corporal (kg) & $78,2( \pm 8,5)$ & $68-95$ & & $60,3( \pm 4,4)$ & $49-65$ & $<0,001^{\dagger}$ \\
Altura (cm) & $178,3( \pm 4,4)$ & $170-189$ & & $166,7( \pm 5,1)$ & $155-173$ & $<0,001^{*}$ \\
Peso graso (\%) & $10,6( \pm 2,5)$ & $7-16$ & & $17,6( \pm 2,7)$ & $14-24$ & $<0,001^{*}$ \\
IMC (kg/m²) & $24,5( \pm 1,9)$ & $22-28$ & & $21,7( \pm 1,1)$ & $19-23$ & $<0,001^{*}$ \\
\hline
\end{tabular}

*Prueba t de Student; † Prueba U de Mann-Whitney

Las variables asociadas al volumen de entrenamiento de la muestra se resumen por sexo en la Tabla 2.

Tabla 2. Características de la experiencia y el volumen de entrenamiento por sexo.

\begin{tabular}{|c|c|c|c|c|}
\hline \multirow[b]{2}{*}{ Variable } & \multicolumn{2}{|c|}{ Varones } & \multicolumn{2}{|c|}{ Mujeres } \\
\hline & Media ( $\pm D E)$ & Rango & Media ( $\pm D E)$ & Rango \\
\hline Años experiencia en pádel (años) & $7,3( \pm 3,3)$ & $4-12$ & $7,8( \pm 3,4)$ & $4-14$ \\
\hline Sesiones en pista (horas/semana) & $8,1( \pm 0,3)$ & $8-9$ & $11,2( \pm 3,1)$ & $8-15$ \\
\hline
\end{tabular}

El perfil de los pliegues cutáneos, perímetros musculares y diámetros óseos se muestran en la Tabla 3. El análisis realizado sobre dichas variables reveló valores significativamente superiores en el sexo femenino respecto al masculino en los pliegues tricipital, muslo y pierna $(p<0,001)$.

Tabla 3. Comparación de variables antropométricas según sexo. 
Rev.int.med.cienc.act.fís.deporte - vol. 19 - número 74 - ISSN: 1577-0354

\begin{tabular}{|c|c|c|c|c|c|}
\hline \multirow[b]{2}{*}{ Pliegues (mm) } & \multicolumn{2}{|c|}{ Varones } & \multicolumn{2}{|c|}{ Mujeres } & \multirow{2}{*}{$\begin{array}{c}\mathbf{p} \\
\text { valor }\end{array}$} \\
\hline & Media (DE) & Rango & Media (DE) & Rango & \\
\hline Bicipital & $4,1( \pm 1)$ & $2,8-6,2$ & $5,2( \pm 10)$ & $3,2-8,2$ & $0,782^{*}$ \\
\hline Tricipital & $9,9( \pm 3,1)$ & $6,2-16$ & $16( \pm 4)$ & $10-22$ & $<0,001^{*}$ \\
\hline Subescapular & $10( \pm 3,7)$ & $5,8-17,4$ & $9,4( \pm 2,7)$ & $6,2-16$ & $0,664^{\dagger}$ \\
\hline Suprailíaco & $10( \pm 4,8)$ & $5,6-21$ & $11,1( \pm 4)$ & $6,2-22$ & $0,176^{\dagger}$ \\
\hline Supraespinal & $9,3( \pm 4,2)$ & $5,6-21$ & $10( \pm 2,7)$ & $6,2-13,8$ & $0,974^{*}$ \\
\hline Abdominal & $17,4( \pm 8,9)$ & $7-38$ & $17,9( \pm 6,6)$ & $8,2-35$ & $0,873^{+}$ \\
\hline Muslo & $15,3( \pm 5,5)$ & $9-26$ & $23( \pm 4,6)$ & $15,4-29,4$ & $<0,001^{*}$ \\
\hline Pierna & $9,5( \pm 4,3)$ & $5-19,4$ & $15,1( \pm 3,9)$ & $9-20$ & $<0,001^{\dagger}$ \\
\hline$\Sigma 6$ pliegues & $72,4( \pm 26,9)$ & $41,8-129,6$ & $92,6( \pm 18,8)$ & $66,8-142,2$ & $<0,02^{*}$ \\
\hline \multicolumn{6}{|l|}{ Perímetros (cm) } \\
\hline Brazo flexionado & $34,4( \pm 2,3)$ & $31,1-39,3$ & $28( \pm 1,2)$ & $25-29,8$ & $<0,001^{\dagger}$ \\
\hline Muslo medio & $56,8( \pm 4,1)$ & $51,1-64,5$ & $51,9( \pm 1,6)$ & $49,1-54,3$ & $<0,001^{\dagger}$ \\
\hline Pierna & $38,4( \pm 2,8)$ & $35-44,5$ & $35,6( \pm 1,6)$ & $33,1-38,4$ & $<0,03^{\dagger}$ \\
\hline \multicolumn{6}{|l|}{ Diámetros (cm) } \\
\hline Biepicondileo & $7,1( \pm 0,3)$ & $6,7-8$ & $6,4( \pm 1,3)$ & $5,8-6,8$ & $<0,001^{*}$ \\
\hline Biepicondiliano & $9,7( \pm 0,4)$ & $9,1-10,7$ & $8,8( \pm 0,4)$ & $8,2-9,4$ & $<0,001^{*}$ \\
\hline Biestiloideo & $5,8( \pm 0,3)$ & $5,5-6,5$ & $4,9( \pm 0,2)$ & $4,7-5,5$ & $<0,001^{*}$ \\
\hline
\end{tabular}

*Prueba t de Student; † Prueba U de Mann-Whitney.

El sumatorio de los seis pliegues (Figura 1) es de 72,4 mm y $92,6 \mathrm{~mm}$ en hombres y mujeres respectivamente $(p<0,02)$. En los varones el componente graso se distribuyó en un $51,8 \%$ para el tronco $(37,4 \mathrm{~mm})$ y un $48,1 \%$ para los miembros $(34,7 \mathrm{~mm})$, mientras que en las mujeres esta relación se encuentra más acusada en los miembros con un $58,4 \%(54,1 \mathrm{~mm})$ del componente graso respecto a los valores del tronco $(41,5 \%$ y $38,4 \mathrm{~mm})$. 


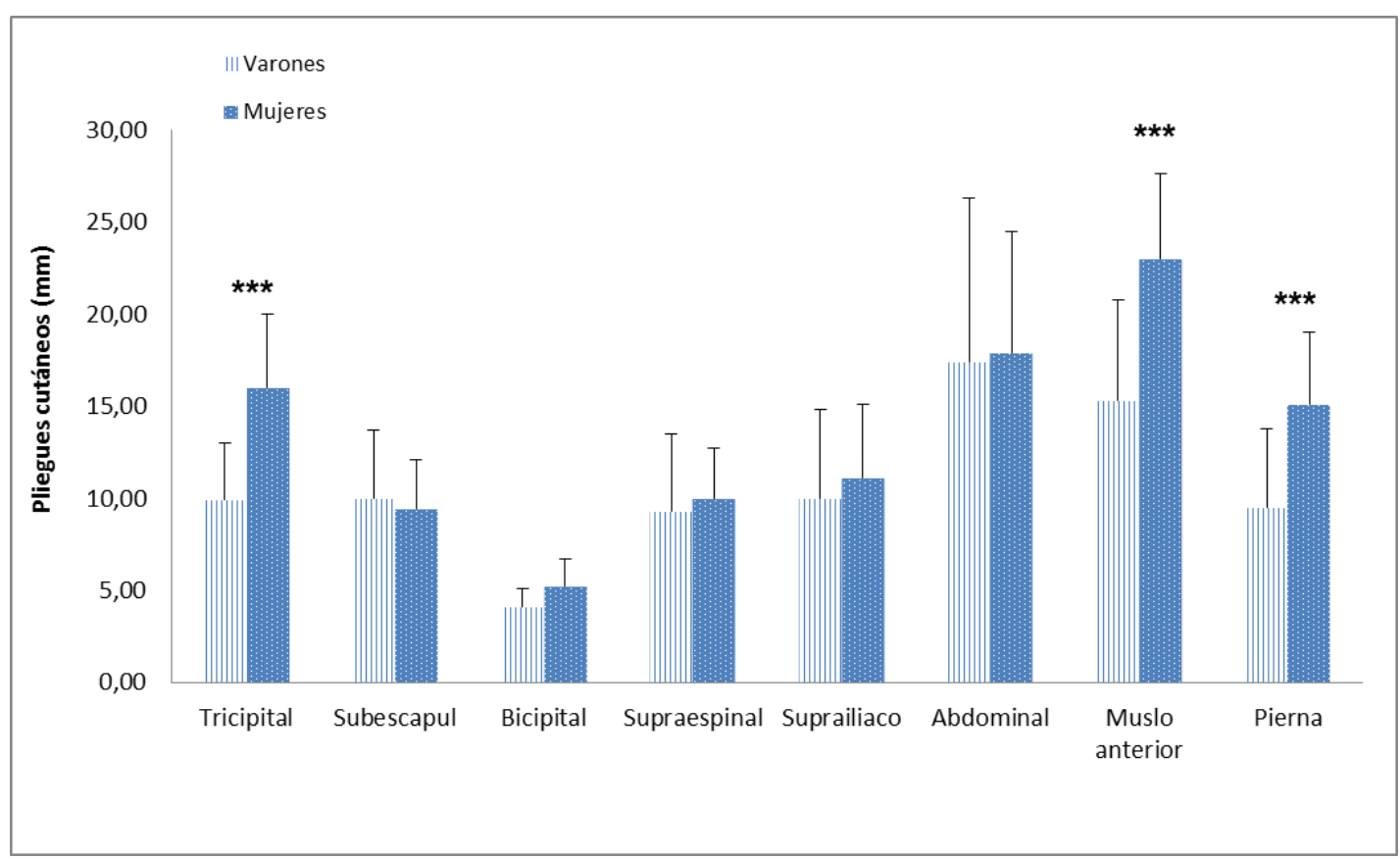

Figura 1. Comparación de pliegues cutáneos de los jugadores de pádel según sexo $\left({ }^{* \star *} p<0,001\right)$.

Los datos obtenidos para la presentación de la composición corporal (Tabla 4) derivan de las categorías propuestas por Carter \& Heath (1990). Los porcentajes de peso muscular en los jugadores son mayores que en las jugadoras $(p<0,001)$ y los de peso graso menores $(p<0,001)$. Los jugadores de pádel de ambos sexos se caracterizan por disponer de una mesomorfía dominante $(5,4 \pm 0,9$ vs $4,0 \pm 0,7)$ y una endomorfía $(2,8 \pm 0,9$ vs $3,8 \pm 0,7)$ mayor que la ectomorfía $(2,0 \pm 0,7$ vs $2,6 \pm 0,6)$. Se ha encontrado una significación estadística entre sexos en los componentes endomórfico $(p<0,01)$, mesomórfico $(p<0,001)$ y ectomórfico $(p<0,05)$.

Tabla 4. Comparación de composición corporal según sexo.

\begin{tabular}{|c|c|c|c|c|c|}
\hline \multirow[b]{2}{*}{ Variable } & \multicolumn{2}{|c|}{ Varones } & \multicolumn{2}{|c|}{ Mujeres } & \multirow{2}{*}{$\begin{array}{c}\mathbf{p}^{*} \\
\text { valor }\end{array}$} \\
\hline & Media (DE) & Rango & Media (DE) & Rango & \\
\hline$\%$ Peso graso & $10,6( \pm 2,5)$ & $7,7-16,2$ & $17,6( \pm 2,7)$ & $14,1-24,9$ & $<0,001$ \\
\hline$\%$ Peso muscular & $43,4( \pm 2,4)$ & $39,4-48,7$ & $36,6( \pm 2,8)$ & $32,7-42,5$ & $<0,001$ \\
\hline \% Peso óseo & $15,8( \pm 0,8)$ & $14,4-17,4$ & $15,5( \pm 0,6)$ & $14,5-16,4$ & 0,328 \\
\hline Endomorfía & $2,86( \pm 0,9)$ & $1,6-5,2$ & $3,8( \pm 0,7)$ & $2,7-5,9$ & $<0,01$ \\
\hline Mesomorfía & $5,47( \pm 0,9)$ & $3,2-7,6$ & $4,0( \pm 0,7)$ & $2,8-5,3$ & $<0,001$ \\
\hline Ectomorfía & $2,02( \pm 0,7)$ & $1,0-3,1$ & $2,6( \pm 0,6)$ & $1,6-3,5$ & $<0,05$ \\
\hline
\end{tabular}

Se han hallado diferencias estadísticamente significativas en el porcentaje de peso graso y muscular $(p<0,001)$ al comparar a los jugadores por sexo. Se aprecia un incremento notable en el porcentaje graso del 10,6 al 17,6\% y un 
decremento del 43,4 al $36,6 \%$ en el componente muscular entre hombres y mujeres, respectivamente.

El somatotipo medio obtenido por los jugadores fue de 2,8-5,4-2,0 (Figura 2) mientras que el de las jugadoras fue de 3,8-4,0-2,6 (Figura 3).

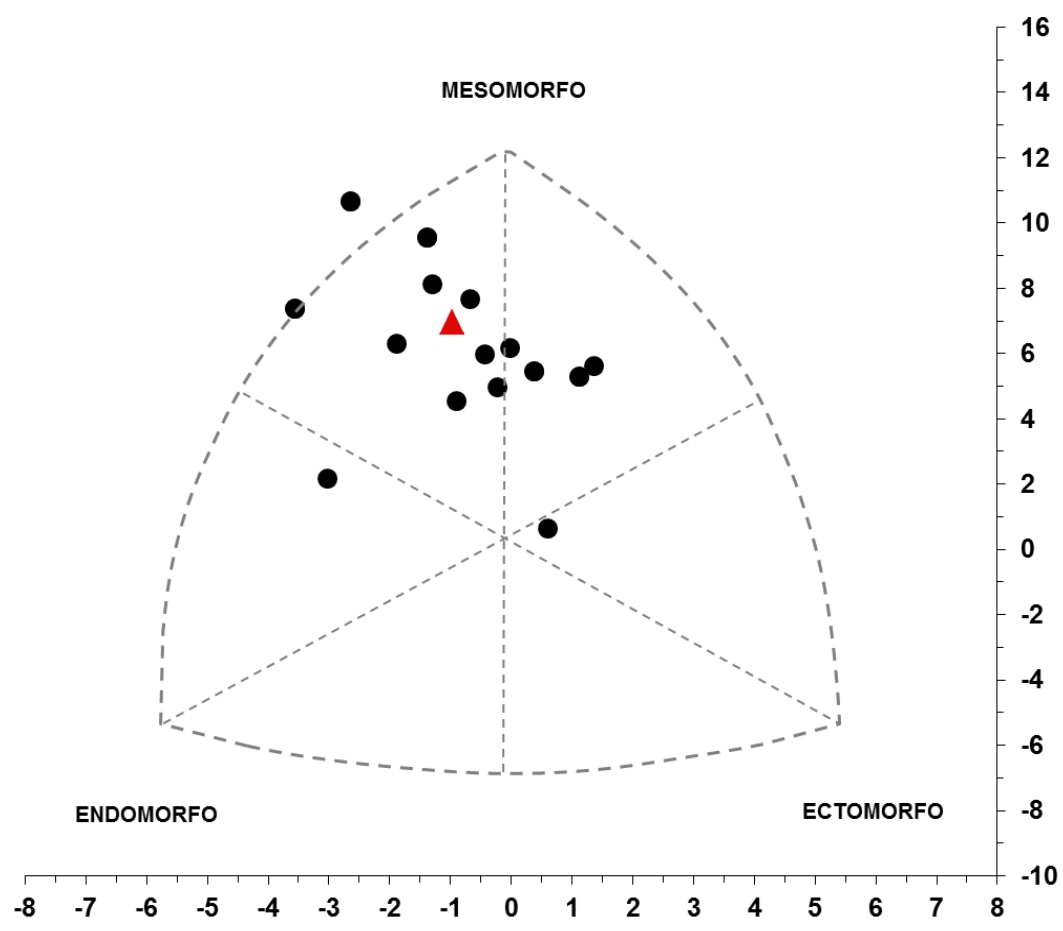

Figura 2. Distribución del somatotipo masculino en la somatocarta

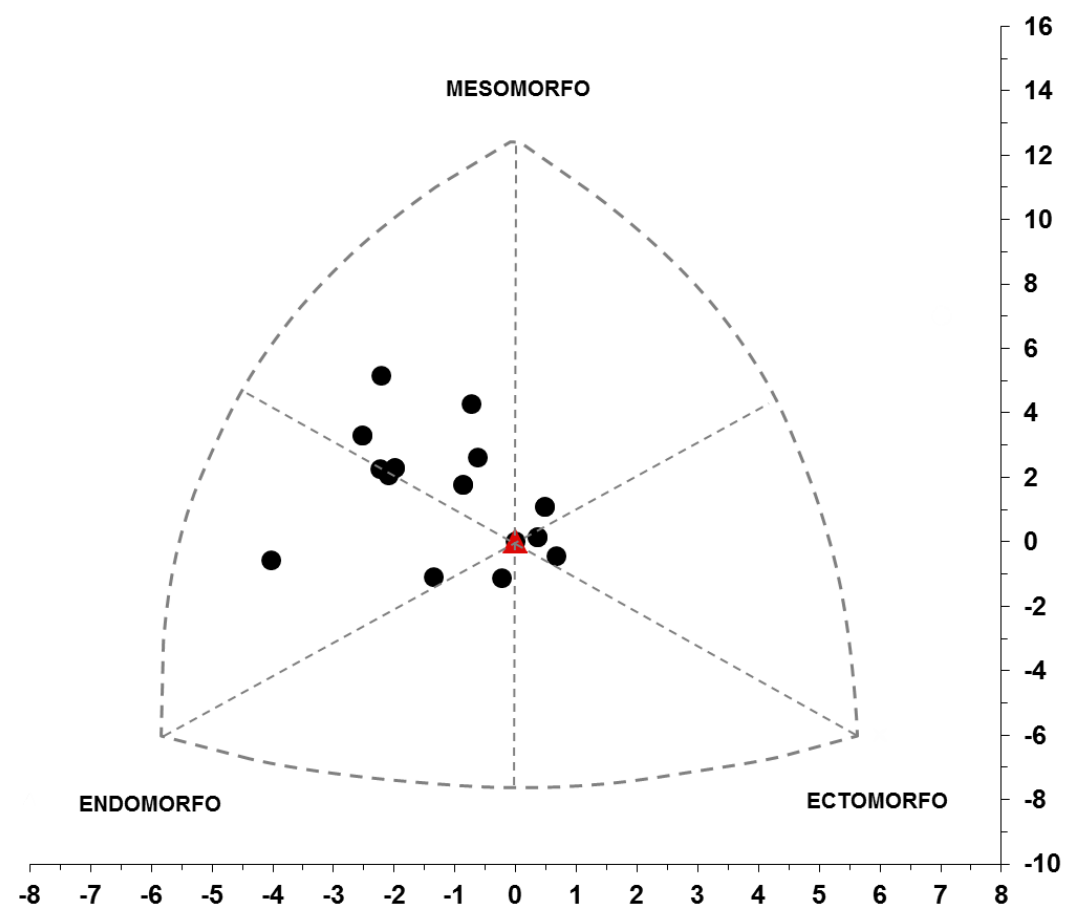

Figura 3. Distribución del somatotipo femenino en la somatocarta. 


\section{DISCUSIÓN}

En la actualidad, el pádel es una disciplina deportiva que se encuentra en pleno periodo de expansión, evolución y desarrollo, por lo que continúa siendo un deporte bastante desconocido, en especial en lo que se refiere a los indicadores biotipológicos que lo caracterizan. Con este estudio se pretende mejorar el conocimiento sobre las características antropométricas y somatotipo de jugadores de pádel de élite de ambos sexos.

Las características antropométricas analizadas en esta investigación pusieron de manifiesto diferencias entre sexos. Los hombres presentan valores superiores a las mujeres en la masa corporal ( 78,2 vs. $60,3 \mathrm{~kg})$, talla (178,3 vs. $166,7 \mathrm{~cm})$ e IMC (24,5 vs. 21,7). Los datos obtenidos en este estudio por los jugadores para estas tres variables han sido muy similares a los hallados por Castillo-Rodríguez et al. (2014) en jugadores de pádel de nivel nacional y algo superiores a los registrados por Martinez-Rodriguez et al. (2015) en deportistas universitarios.

Si comparamos los datos obtenidos por la muestra masculina con la de otros deportistas en diferentes disciplinas de raqueta y pala, se aprecia que los jugadores de pádel presentan valores de talla ligeramente superiores $(178,3 \pm 4,4$ vs. $177 \pm 0,06)$ a los hallados en tenis de mesa (Pradas et al., 2013), pero inferiores $(178,3 \pm 4,4$ vs. $181 \pm 5,7)$ a los encontrados para el bádminton (Faccini \& Dal Monte, 1996) y el tenis profesional $(184 \pm 7,1)$ (Hornery, Farrow, Mújika, \& Young, 2007). En squash los jugadores de alto nivel analizados presentaron diferentes alturas $172,6 \pm 4,3 \mathrm{~cm}, 174,5 \pm 0,7 \mathrm{~cm}$ y $182 \pm 7 \mathrm{~cm}$ (Chin et al., 1995; Johansen \& Jensen, 1999), que podrían estar relacionadas en cada caso con el propio fenotipo de las poblaciones estudiadas.

En las mujeres los valores encontrados para las variables antropométricas masa, talla e IMC son similares a los obtenidos por Castillo-Rodríguez et al. (2014) en jugadoras españolas de pádel de nivel nacional. Al no existir suficientes estudios sobre este deporte se hace necesario volver a comparar estos resultados con otros deportes de raqueta y pala. Se aprecia que la talla de las jugadoras de pádel es ligeramente superior a la del tenis de mesa (Pradas et al., 2013), e inferior a la del tenis (Hornery et al., 2007), pero se encuentra comprendida dentro del rango de estatura descrita en jugadoras de bádminton (Vicén et al., 2012).

Los valores que presentan los jugadores de pádel en ambos sexos inducen a pensar que la variable altura puede no ser tan importante en este deporte respecto a otras disciplinas como el tenis o el bádminton. Probablemente, una de las posibles explicaciones relacionadas con la estatura puede tener una relación directa con la dinámica de juego que se desarrolla en este deporte. En el pádel se permite la posibilidad de dejar pasar la bola para golpearla tras su bote, impacto y posterior rebote sobre el cerramiento de fondo, por lo que no resulta imprescindible realizar golpeos sobre bolas altas, siendo en este deporte más importante el juego de habilidad que el de potencia, sobre todo para las 
acciones de juego que más se producen, que son aquellas que se desarrollan próximas a la red, siendo consideradas éstas como las de mayor relevancia y trascendencia en el pádel (Courel-lbáñez, Sánchez-Alcaraz, \& Cañas, 2015).

Las jugadoras registraron una masa corporal muy similar a la obtenida por Castillo-Rodríguez et al.(2014) pero más elevada que la encontrada en deportes como el tenis de mesa (Pradas et al., 2013), aunque muy próxima a la registrada en bádminton (Vicén et al., 2012), pero inferior a la hallada en el tenis (Hornery et al., 2007).

La muestra masculina presentó una masa corporal similar a la hallada por Castillo-Rodríguez et al. (2014) en jugadores de pádel de nivel nacional, y algo superior $(78,2 \pm 8,5$ vs. $74,3 \pm 8,6)$ respecto a jugadores universitarios (MartinezRodriguez et al., 2015). En la comparativa con otros deportes de raqueta y pala los jugadores analizados han mostrado una masa corporal mayor. En tenis de mesa se hallaron valores de 73,6 55,6 y $71,9 \pm 9,1$ en jugadores de élite de la selección chilena y española respectivamente (Pradas et al., 2013; Sepúlveda et al., 2015). Son igualmente más pesados que jugadores de squash analizados por Chin et al. (1995), con valores de $67,7 \pm 6,9 \mathrm{~kg}$. En la comparativa con jugadores de tenis investigados por Ooi et al. (2009), la muestra analizada también ha mostrado valores mayores aunque con una menor diferencia de peso.

Este mayor peso registrado en jugadores de pádel respecto a otros deportes de raqueta y pala podría encontrarse estrechamente relacionado con la intensidad a la que se desarrolla este deporte, siendo inferior a disciplinas como el bádminton, tenis, squash o tenis de mesa, en donde las acciones de juego que se producen se realizan a una elevada velocidad (Cabello-Manrique \& Gonzalez-Badillo, 2003; Chin et al., 1995; Fernández et al., 2006; Pradas et al., 2013). En este sentido, un peso corporal excesivo en un jugador de pádel, en particular del componente adiposo, puede generar una clara desventaja frente al adversario, produciéndose un efecto negativo sobre el rendimiento, fundamentalmente para realizar movimientos específicos, como los que se producen en la red o para devolver pelotas muy próximas a las paredes laterales y de fondo, por el mayor desplazamiento de tejido no propulsivo, suponiendo la realización de esfuerzos musculares más severos por el grado de aceleración y desaceleración que requieren (Chin et al., 1995; Pradas et al., 2013).

Entre los pliegues cutáneos analizados, y a pesar de presentar valores superiores las mujeres en siete de los ocho pliegues medidos, sólo se han encontrado diferencias significativas en tres de ellos y correspondientes a las extremidades.

Las diferencias halladas entre sexos en las variables antropométricas masa, talla y en el IMC, así como en los diferentes perímetros y diámetros evaluados, son la consecuencia de un marcado dimorfismo sexual existente entre hombres y mujeres (Cox \& Calsbeek, 2010). 
El porcentaje de peso graso (PG) obtenido fue inferior en los hombres respecto a las mujeres, de manera similar a lo descrito en la literatura para diferentes deportes de raqueta y pala (Pradas et al., 2013). Martinez-Rodriguez et al., (2015) describen valores de PG en jugadores de pádel universitarios muy por encima a los encontrados en los jugadores de elite analizados en esta investigación. Estos resultados ponen de manifiesto que los porcentajes de PG son inferiores cuanto mayor es el nivel de rendimiento de los jugadores.

Cuando comparamos el PG de la muestra masculina con los estudios existentes sobre deportes de raqueta y pala, se aprecia que los jugadores de pádel son los deportistas que presentan un menor PG. Pradas et al. (2013) encontró valores de $P G$ en varones de alto nivel de tenis de mesa de $12 \pm 2,7 \%$. En bádminton Majumdar (1997) y Ooi et al. (2009) obtuvieron valores ligeramente superiores al $12 \%$. En squash se han encontrado valores de PG en jugadores de alto nivel inferiores al 12\% (Chin et al., 1995).

No se han encontrado datos relativos al PG en jugadoras de pádel en la literatura consultada. Los valores obtenidos en este estudio $(17,6 \pm 2,7 \%)$ son superiores a los hallados por (Pradas et al., 2013) en jugadoras de tenis de mesa de elite $(14,7 \pm 1,5 \%)$, pero ligeramente inferiores a los hallados en jugadoras de bádminton y tenis con valores de $23,6 \pm 3,3 \%$ y $18,1 \pm 2,3 \%$ respectivamente (Hughes, Reilly, Hughes, \& Lees, 1995; Pyke, Elliott, \& Pyke, 1974). EI PG corporal puede considerarse en el jugador de pádel como un factor que puede limitar su rendimiento. Además, tal y como apunta Chin et al. (1995), un exceso de grasa corporal podría aumentar el gasto de energía que se requiere durante una competición, provocando un impacto negativo sobre el rendimiento físico y técnico-táctico que se va acentuando a medida que transcurre el partido.

El análisis del somatotipo en los jugadores de pádel de alto nivel revela en los hombres un tipo mesomorfo-endomórfico, muy parecido al hallado en otras investigaciones de índole similar (Castillo-Rodríguez et al., 2014; MartinezRodriguez et al., 2015), en donde el componente mesomórfico es el predominante, pero existiendo diferencias importantes en los valores referentes al componente endomórfico, probablemente vinculadas al mayor nivel técnico y físico de la muestra de alto nivel analizada.

En análisis del somatotipo revela una distribución amplia de los jugadores por toda la somatocarta y específicamente por la zona mesomórfica. Sin embargo, las mujeres se sitúan más en la zona central y en el sector endomesomórfico de manera parecida a las jugadoras de tenis (Solanellas, Tuda, \& Rodríguez, 1996).

Aunque la mesomorfía es el componente principal en ambos sexos, los resultados de la composición corporal muestran un mayor porcentaje de peso graso en mujeres y un mayor porcentaje de peso muscular y óseo en los hombres, siendo estas diferencias sexuales habituales entre hombres y mujeres (Cox \& Calsbeek, 2010). 
No ha sido posible realizar una comparación de los somatotipos obtenidos al no existir en la actualidad datos sobre un somatotipo de referencia en el deporte del pádel.

\section{CONCLUSIONES}

La altura no parece ser una variable tan importante en este deporte respecto a otros deportes de raqueta.

Los pliegues tricipital, muslo y pierna son significativamente inferiores en los hombres.

El componente adiposo, expresado tanto en porcentajes como en el sumatorio de seis pliegues cutáneos, presenta valores significativamente superiores en mujeres.

La composición corporal en este deporte presenta en ambos sexos una mesomorfía dominante y una endomorfía mayor que la ectomorfía.

El somatotipo obtenido por los hombres es mesomorfo-endomórfico mientras que el de las mujeres es endomorfo-mesomorfo.

\section{REFERENCIAS BIBLIOGRÁFICAS}

Cabello-Manrique, D., \& Gonzalez-Badillo, J. J. (2003). Analysis of the characteristics of competitive badminton. British Journal of Sports Medicine, 37(1), 62-66. https://doi.org/10.1136/bjsm.37.1.62

Carter, J. E. L., \& Heath, B. H. (1990). Somatotyping: development and applications (Vol. 5). Cambridge University Press.

Castellar, C., Pradas, F., Quintas, A., Arraco, S., \& Pérez, J. B. (2015). Perfil condicional de jugadoras de pádel de élite. Revista Andaluza de Medicina Del Deporte, 8(4), 185. https://doi.org/10.1016/j.ramd.2015.08.004

Castillo-Rodríguez, A., Hernández-Mendo, A., \& Alvero-Cruz, J. R. (2014). Morphology of the elite paddle player - Comparison with other racket sports. International Journal of Morphology, 32(1), 177-182. http://doi.org/10.4067/S0717-950220140001000030

Chin, M. K., Steininger, K., So, R. C., Clark, C. R., \& Wong, A. S. (1995). Physiological profiles and sport specific fitness of Asian elite squash players. British Journal of Sports Medicine, 29(3), 158-164. https://doi.org/10.1136/bjsm.29.3.158

Courel-Ibáñez, J., Sánchez-Alcaraz, B. J., \& Cañas, J. (2015). Effectiveness at the net as a predictor of final match outcome in professional padel players. International Journal of Performance Analysis in Sport, 15(2), 632-640. https://doi.org/10.1080/24748668.2015.11868820

Cox, R. M., \& Calsbeek, R. (2010). Sex-specific selection and intraspecific variation in sexual size dimorphism. Evolution, 64(3), 798-809. http://doi.org/10.1111/j.1558-5646.2009.00851.x 
de Hoyo, M., Sañudo, B., \& Carrasco, L. (2007). Demandas fisiológicas de la competición en pádel. Revista Internacional de Ciencias Del Deporte, 3(8), 53-58. https://doi.org/10.5232/ricyde2007.00805

Faccini, P., \& Dal Monte, A. (1996). Physiologic demands of badminton match play. American Journal of Sports Medicine, 24(SUPPL.), S64-S66. https://doi.org/10.1177/036354659602406S19

Fernández, J., Mendez-Villanueva, A., \& Pluim, B. M. (2006). Intensity of tennis match play. British Journal of Sports Medicine, 40(5), 387-391. http://doi.org/10.1136/bjsm.2005.023168

Hornery, D. J., Farrow, D., Mújika, I., \& Young, W. (2007). An integrated physiological and performance profile of professional tennis. British Journal of Sports Medicine, 41(8), 531-536. http://doi.org/10.1136/bjsm.2006.031351

Hughes, M. G., Reilly, T., Hughes, M., \& Lees, A. (1995). Physiological demands of training in elite badminton players. Et Al. Science and Racket Sports, London, 32-37.

Johansen, L., \& Jensen, K. (1999). Physical capacity of Danish elite squash players. Medicine and Science in Sports and Exercise, 31(5), S256. https://doi.org/10.1097/00005768-199905001-01233

Majumdar, P. (1997). Physiological analysis to quantify training load in badminton. British Journal of Sports Medicine, 31(4), 342-345. https://doi.org/10.1136/bjsm.31.4.342

Martinez-Rodriguez, A., Collado, E. R., \& Vicente-Salar, N. (2015). Body composition assessment of paddle and tennis adult male players. Nutricion Hospitalaria, 31(3), 1294-1301. http://doi.org/10.3305/nh.2015.31.3.8004

Mellor, S., Hughes, M. D., Reilly, T., Robertson, K., Reilly, T., Hughes, M., \& Lees, A. (1995). Physiological profiles of squash players of different standards. In T. Reilly, M. D. Hughes, \& A. Lees (Eds.), Science and Racket Sports (pp. 72-75). London: E \& FN Spon.

Ooi, C. H., Tan, A., Ahmad, A., Kwong, K. W., Sompong, R., Ghazali, K. A. M., ... Thompson, M. W. (2009). Physiological characteristics of elite and subelite badminton players. Journal of Sports Sciences, 27(14), 1591-1599. http://doi.org/10.1080/02640410903352907

Pradas, F., Cachón, J., Otín, D., Quintas, A., Inmaculada, S., \& Castellar, C. (2014). Anthropometric, physiological and temporal analysis in elite female paddle players. Retos: Nuevas Tendencias En Educación Física, Deporte Y Recreación, 2041(25), 107-112.

Pradas, F., González-Jurado, J. A., Molina Sotomayor, E., \& Castellar Otín, C. (2013). Anthropometric characteristics, body composition and somatotype of high level table tennis players. International Journal of Morphology, 31(4), 1355-1364. http://doi.org/10.4067/S0717-95022013000400033

Pyke, S., Elliott, C., \& Pyke, E. (1974). Performance testing of tennis and squash players. British Journal of Sports Medicine, 8(2-3), 80. https://doi.org/10.1136/bjsm.8.2-3.80

Sánchez-Muñoz, C., Sanz, D., \& Zabala, M. (2007). Anthropometric characteristics, body composition and somatotype of elite junior tennis players. British Journal of Sports Medicine, 41(11), 793-799. https://doi.org/10.1136/bjsm.2007.037119 
Sepúlveda, R. Y., Barraza, F., Soto, G. R., Báez, E., \& Tuesta, M. (2015). Anthropometric chilean table tennis players of competitive features. Nutricion Hospitalaria, 32(4), 1689-1694. http://doi.org/10.3305/nh.2015.32.4.9547

Solanellas, F., Tuda, M., \& Rodríguez, F. A. (1996). Valoración cineantropométrica de tenistas de diferentes categorías. Apunts, 44-45.

Vicén, P. A., Abián-Vicén, J., \& Sampedro, J. (2012). Anthropometric analysis of body symmetry in badminton players. International Journal of Morphology, 30(3), 945-951. http://doi.org/10.4067/S0717-95022012000300030

Withers, R. T., Craig, N. P., Bourdon, P. C., \& Norton, K. I. (1987). Relative body fat and anthropometric prediction of body density of male athletes. European Journal of Applied Physiology and Occupational Physiology, 56(2), 191-200. https://doi.org/10.1007/BF00640643

Número de citas totales / Total references: 25 (100\%)

Número de citas propias de la revista / Journal's own references: $0(0 \%)$

Rev.int.med.cienc.act.fís.deporte - vol. 19 - número 74 - ISSN: 1577-0354 